\title{
TITLE: RATE-DEPENDENT SPALLATION PROPERTIES OF TANTALUM
}

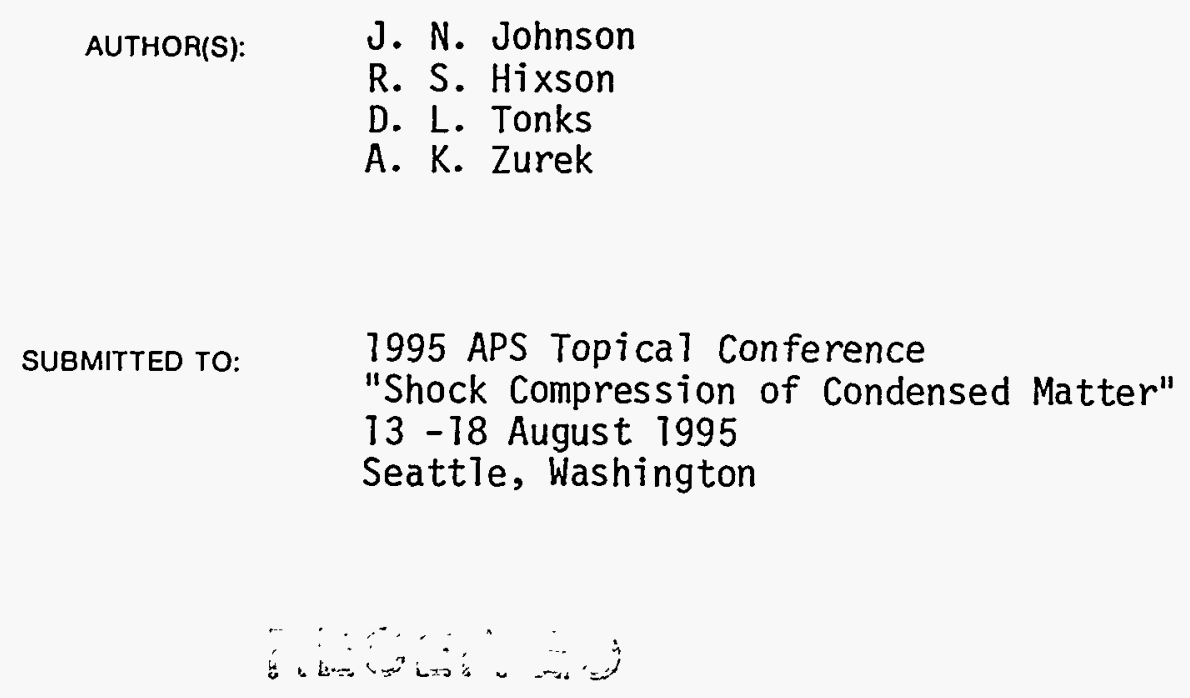

A19629095

09 


\section{DISCLAIMER}

Portions of this document may be illegible in electronic image products. Images are produced from the best available original document. 
This report was prepared as an account of work sponsored by an agency of the United States Government. Neither the United States Government nor any agency thereof, nor any of their employees, makes any warranty, express or implied, or assumes any legal liability or responsibility for the accuracy, completeness, or usefulness of any information, apparatus, product, or process disclosed, or represents that its use would not infringe privately owned rights. Reference herein to any specific commercial product, process, or service by trade name, trademark, manufacturer, or otherwise does not necessarily constitute or imply its endorsement, recommendation, or favoring by the United States Government or any agency thereof. The views and opinions of authors expressed herein do not necessarily state or reflect those of the United States Government or any agency thereof.

\title{
RATE-DEPENDENT SPALLATION PROPERTIES OF TANTALUM
}

\author{
J.N. Johnson, R.S. Hixson, D.L. Tonks, and A.K. Zurek \\ Los Alamos National Laboratory, Los Alamos, NM, 87545, USA
}

\begin{abstract}
Spallation experiments are conducted on high-purity tantalum using VISAR instrumentation for impact stresses of 9.5 GPa and 6.0 GPa. The high-amplitude experiment exhibits very rapid initial spall separation, while the low-amplitude shot is only slightly above the threshold for void growth and thus exhibits distinct rate-dependent spallation behavior. These experiments are analyzed in terms of simple tensile fracture criteria, a standard rate-dependent void-growth model, and a rate-dependent void growth model in which the expected plastic volume strain makes no contribution to the relaxation of the mean stress. Recovery tests and VISAR measurements suggest an additional resistance to spallation that follows the rapid coalescence of voids; this effect is termed the secondary spall resistance and is due to the convoluted nature of the spall plane and the resulting interlocking fracture pattern that is developed and for which the stress remains urrelieved until the spall planes have separated several hundred microns.
\end{abstract}

\section{INTRODUCTION}

Spallation in metals remains an important research subject in the field of shock compression science in spite of the wealth of existing spallation models and corresponding data. Spallation, like dynamic plasticity, is controlled by defects rather than the perfect lattice and hence substantial spall strength variation with impact amplitude and strain rate is sometimes seen where none is expected. In the case of tantalum (bcc) the plastic flow properties are controlled by the Peierls stress (inherent to the perfect lattice) and are not exceedingly sensitive to imperfections. The spall strength, however, is controlled by large-scale impurities that either provide initial porosity or act as nucleation sites for voids. Simple tensile fracture models are often unable to adequately represent material spall behavior because of the strong dependence on ratedependent properties associated with void initiation, growth, and coalescence.

In this work we present a study of the spallation properties of tantalum (i) just above the spallation threshold and (ii) at an impact stress one and onehalf times the spallation threshold. The experimental measurements are compared with those of Isbell, et al (1). The new results show the dependence of spall strength on impact amplitude and some of the complexities of the spallation process near threshold conditions when analyzed in detail in terms of void-growth models. A new phenomenon termed "secondary spall resistance" is observed and described theoretically.

\section{EXPERIMENT}

Shock-wave experiments are performed with a 50-mm-diameter gas gun. Projectile velocity and tilt are measured immediately before impact by means of a stepped circular array of shorting pins surrounding the target disk. For these experiments, tilts are typically $1.0-1.5 \mathrm{mrad}$, and impact velocities range from approximately 200 to $300 \mathrm{~m} / \mathrm{s}$ : these velocities produce longitudinal stresses in the range of 6.0 to $9.5 \mathrm{GPa}$ for symmetric impact.

A shock-release profile is measured using a push/pull VISAR (2) with a sapphire window. Spallation experiments are conducted with stressfree back surfaces, also with VISAR instrumentation.

The chemical composition of this material is as follows (in $\mathrm{ppm}$ ): $\mathrm{C}(6), \mathrm{O}(56), \mathrm{N}(24), \mathrm{H}(<1)$, $\mathrm{Fe}(19), \mathrm{Ni}(25), \mathrm{Cr}(9), \mathrm{W}(41), \mathrm{Nb}(26), \mathrm{Ta}($ balance).

\section{Calculation}

Following the establishment of the shock/release behavior $(3,4)$ in the absence of spallation, a set of 


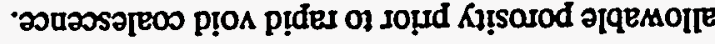

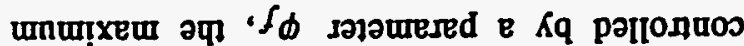

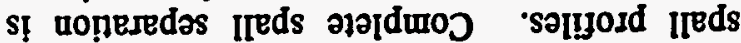

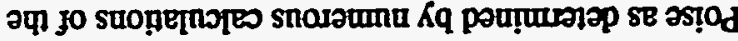

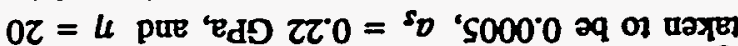

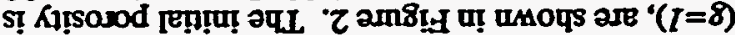

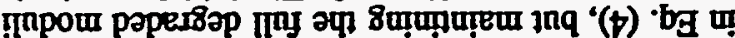

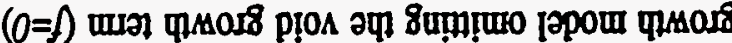

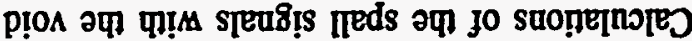

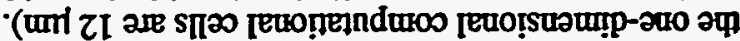

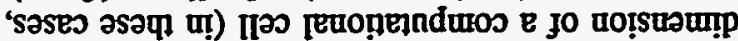

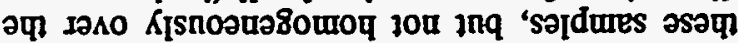

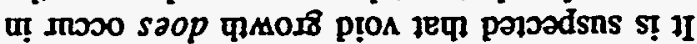

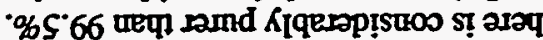

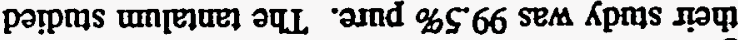

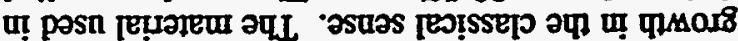

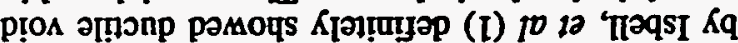

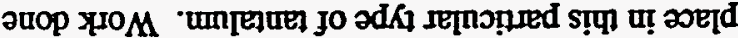

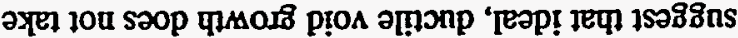

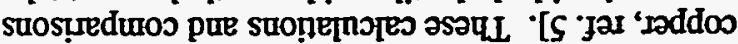

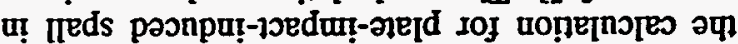

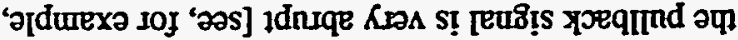

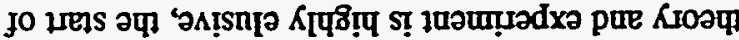

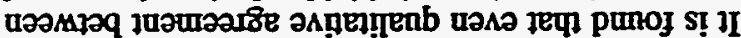

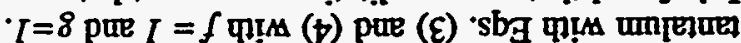

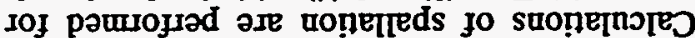

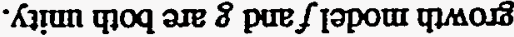

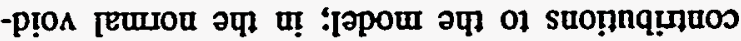
snouter joguos of pasn are 8 pure $f$ sannutuenb әqL

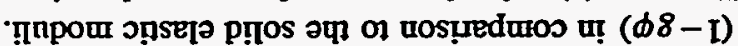

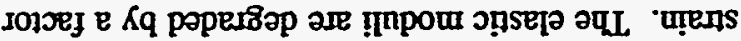

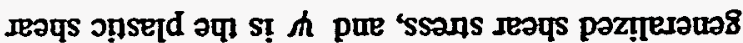

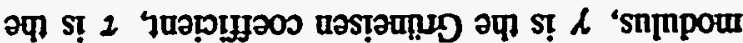

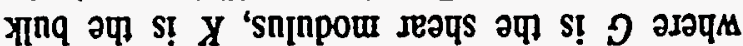

$$
(\phi-I) / \phi f(\underline{d} \ell-X)+\not \underline{\phi}(\nu \ell-D) z-=d
$$

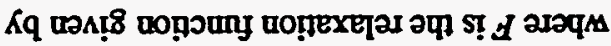

$$
g=(d / \varphi)(\varepsilon / \partial \downarrow+X)-?
$$

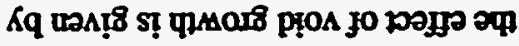

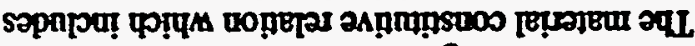
पTMar8 plon joj ssans MOJ

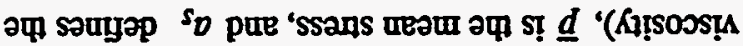

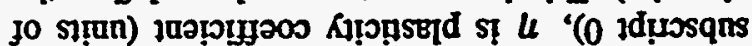

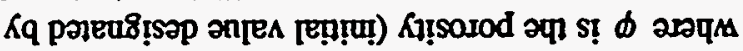

$$
\phi 80 I(\phi-I)^{s} v+\underline{d}=\Phi \nabla
$$

(I)

$$
\phi_{\varepsilon / Z}\left[\frac{\left({ }^{\circ} \phi-I\right)}{(\phi-I)^{\circ} \phi}\right] \frac{u}{\varepsilon / I}-=\phi
$$

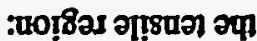
u! אịsolod jo ว8น

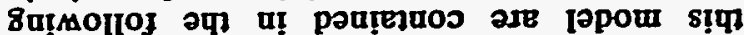

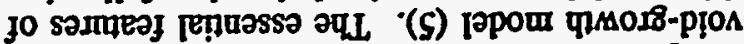

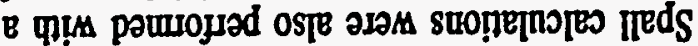

paumoso

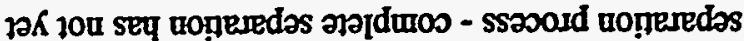

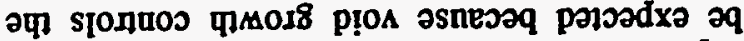

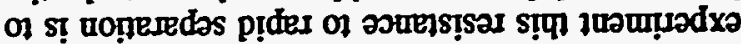

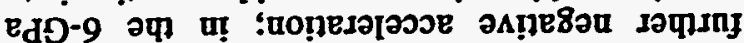

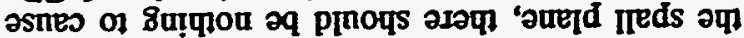

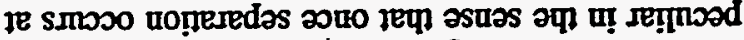

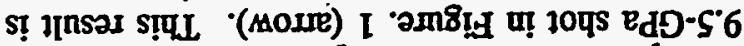

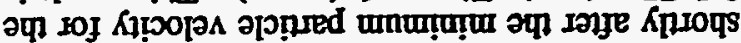

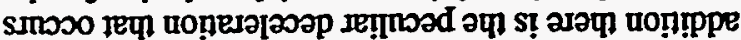

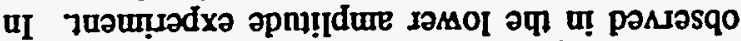

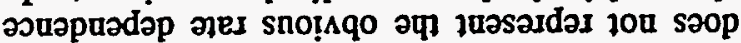

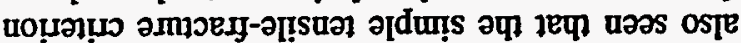

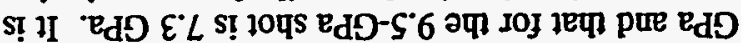

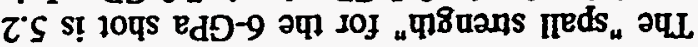

TIreds

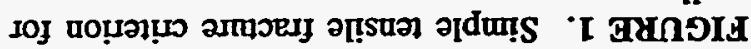

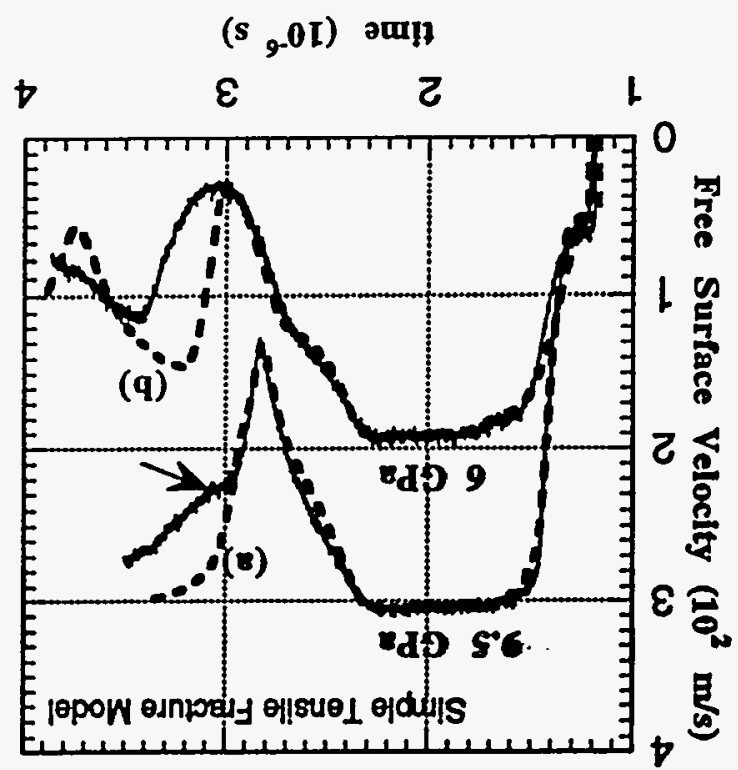

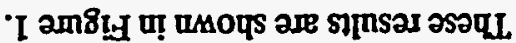

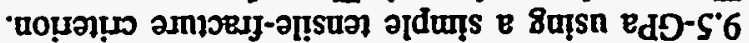
pus -9 yo sassans ioedun le unn[e]uer jo əsuodsax

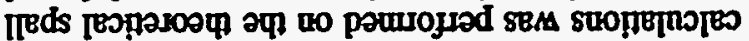




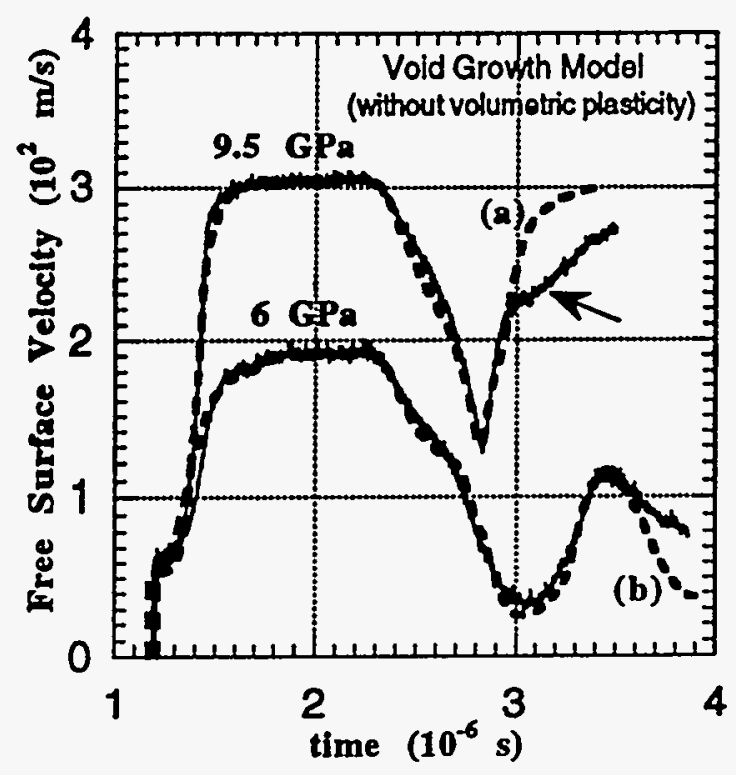

FIGURE 2. Void growth model for spall: (a) $\varphi_{f}=0.30$; (b) $\varphi_{f}=0.43$.

The rate-dependent nature of the spall process for the 6-GPa experiment is obvious. The fracture porosity in this case is $\varphi_{f}=0.43$. For the $9.5-\mathrm{GPa}$ experiment $\varphi_{f}=0.30$, and there is no essential difference between the calculated spall response for the simple tensile fracture criterion and for the voidgrowth model. The fact that the fracture porosities are different is unusual, and is something that requires further investigation.

If the link-up, or void coalescence, step in spallation were simply a rate-dependent plastic flow process, then it would be expected that higher rates of tensile loading would allow greater porosities to be achieved prior to fracture; the opposite is observed here.

The agreement shown in Figure 2 is suggestive of a material for which the void growth is limited to a small region within a computational cell (brittle fracture) and whose moduli are reduced by the presence of this damage, but whose volumetric plastic strain is not strongly affected.

\section{SECONDARY SPALL RESISTANCE}

One of the most interesting and peculiar observations associated with the $9.5 \mathrm{GPa}$ spall signal is the sudden deceleration that occurs in the pull-back signal (arrow, Figure 1). It appears that the material has undergone complete spallation, i.e., material separation, and then finds that there remains a substantial restoring force tending to decelerate the spalled piece. This remained a puzzle until recovery experiments were performed to examine the nature of the actual spall plane. The spall region is shown in Figure 3 for a $10 \mathrm{GPa}$ impact stress.

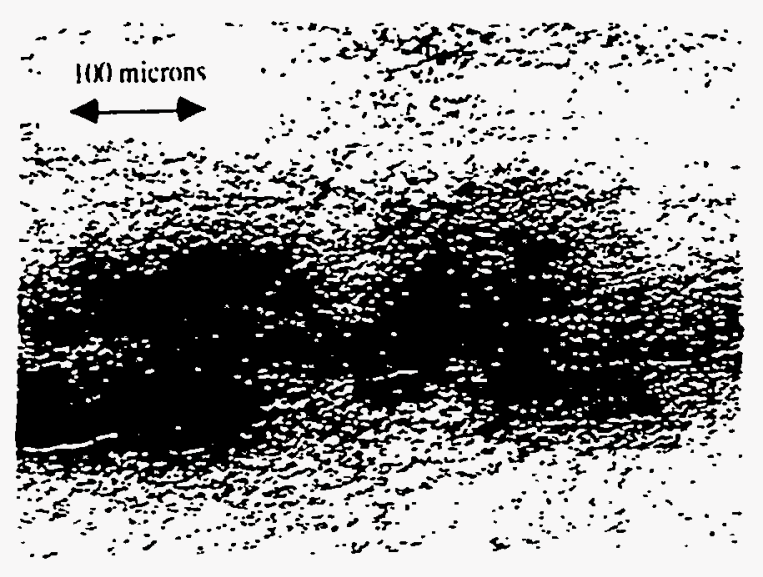

FIGURE 3. Spalled region for peak impact stress of $10 \mathrm{GPa}$.

It is seen from Figure 3 that the spall plane is not a distinct fracture surface, but rather is extended over several tens of microns in the direction of wave propagation (vertical). This observation suggests that the initial loss of strength takes place by the coalescence of voids to form a system of small cracks (long dimension perpendicular to impact direction) extended over a finite region of several tens of microns normal to the spall plane. The initial loss of material strength obtains from the formation and elastic opening of these cracks and the corresponding drop (to near zero) of the longitudinal tensile stress. Following this initial loss of tensile strength, the extended spall plane pulls apart and undergoes additional linking of these cracks to eventually form the separated spall plane, but not before developing considerable secondary resistance to separation: this is what we refer to as secondary spall resistance (SSR)

The SSR is modeled in terms of an additional tensile stress that develops following the usual spallation process. As the separation distance $x$ 
between the left and right sides of the spall surface increases, the SSR is given by

$$
\begin{aligned}
& \sigma_{S S R}=0 \text { for } x<a \text { and } x>b \\
& \sigma_{S S R}=f \sigma_{S}[(x-b) /(b-a)] \text { for } a<x<b
\end{aligned}
$$

where $\sigma_{s}$ is the absolute magnitude of the spall strength and $f$ is a nondimensional number less than unity. Generally $a$ will be on the order of a few microns (the onset of SSR) and $b$ will be on the order of a hundred microns (the end of SSR). Equations (3) represent the stress necessary to pull apart the convoluted spall plane shown in Figure 3.

Calculations of the spallation behavior with this model of SSR is shown in Figure 4 for $a=5$ microns, $b=200$ microns, and $f=0.20$.

\section{DISCUSSION}

The fracture properties of metals is extremely complex. The combination of plastic flow properties and impurity content that control the fracture process provides very subtle differences that result in brittle behavior in some cases and fully ductile behavior in others. Temperature is another parameter that we have not yet even begun to investigate, but one that obviously has a strong influence on these properties.

Tantalum studied previously (1) $(99.5 \%$ pure) exhibited classical ductile spallation properties in the range of impact stresses from approximately 7 $\mathrm{GPa}$ to $9 \mathrm{GPa}$. The material studied here quite obviously fails to behave in the ideal manner and consequently the ductile void-growth model must be modified in order to represent the time-resolve spallation data. It is found empirically that a fit to the data can be obtained by omitting the void growth term from the relaxation function $F, \mathrm{Eq}$. (4). This is clearly not very satisfying and other methods were sought to obtain a similar effect. A second method was found in which the factors $f$ and $\&$ (multiplying the void growth term in the relaxation function and in the damage term controlling the moduli) were equal and less than unity. This also gives a good fit to the experimental data.

A new effect was observed in the $9.5 \mathrm{GPa}$ experiment described here. This termed Secondary Spall Resistance (SSR) and has to do with the resistance provided by the extended spall plane as it tries to pull apart in the fashion of a jigsaw puzzle.

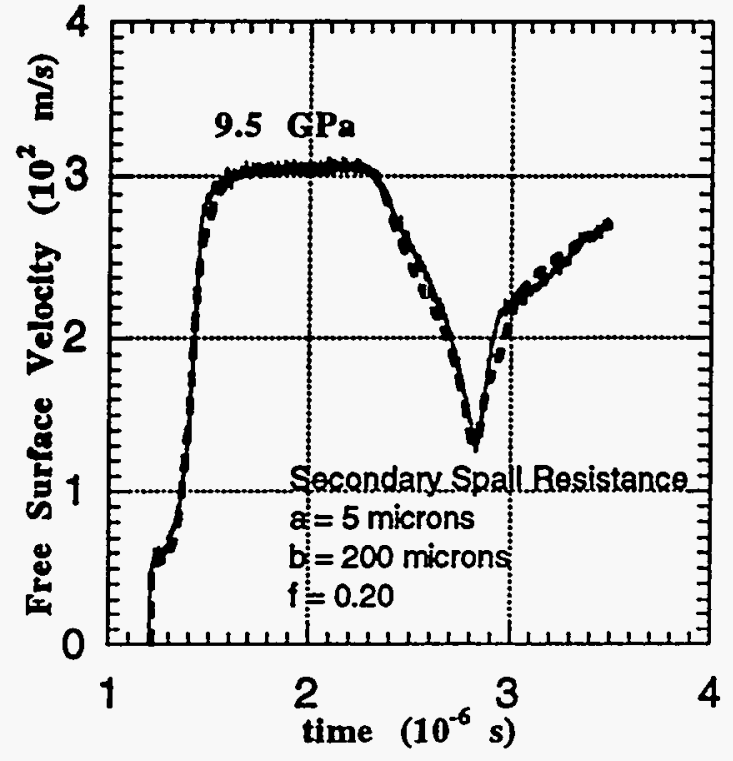

FIGURE 4. Calculation of spall signal with secondary spall resistance included.

This effect is very pronounced, and is represented in terms of a secondary force that applies once the (left and right) spall surfaces have moved apart a few microns and continue until separation reaches a few hundred microns.

\section{REFERENCES}

1. Isbell, W.M., Christman, D.R., and Babcock, S.G., "Measurement of Dynamic Properties of Muterials, Vol. VI, Tantalum," Defense Nuclear Agency Repor DASA 2501-6, February (1972).

2. Hemsing, W.F., "Velocity Sensing Interferometer (VISAR) Modification," Rev. Sci hustrum. 50, 73-78 (1979).

3. Johnson, J.N., Hixson, R.S., Gray III, G.T., and Morris, C.E., "Quasielastic Release in Shock-Compressed Solids," J. Appl. Phys. 72, 429-441 (1992).

4. Johnson, J.N., Hixson, R. S., Tonks, D. L, and Gray III, G.T., "Shock-Compression and Quasielestic Release in Tantahum," Hiph Bressure Science und Technology - 1993 edited by S. C. Schmidt, J. W. Shaner, G. A. Samara, and M. Ross, American Institute of Physic Conference Proceedings 309, Part 2, 1095 1098 (1993).

5. Johnson, J.N., "Dynamic Fracturo and Spallation is Ductile Solids," J. Appl. Phys. 52, 2812-2825 (1981). 